\title{
EDITORIAL
}

\section{How can we improve our assessment of inflammation in clinical studies of COPD that use bronchial biopsies?}

\author{
Q. Hamid
}

I $\mathrm{n}$ this issue of the European Respiratory Journal, GAMBLE et al. [1] have retrospectively examined the sources of biological variability in the quantification of inflammatory cells in endobronchial biopsies using immunostained samples. The samples were taken from 51 subjects with chronic obstructive pulmonary disease (COPD) that were obtained from a clinical trial published by the same group. They reported that the distribution of variance contributed by different sources was similar for different inflammatory cell types. Using immunocytochemistry for CD8+ cells, the largest contribution to intersubject variability was time between biopsies of the placebo-treated group followed by airway generation, biopsy, zone and section. They concluded that examining one section of one biopsy from each of two airway generations would require a sample size of 32 subjects per group to show a difference of one doubling or halving in CD8+ cells. This would compare with a group of 47 subjects, if only one airway generation was sampled. They suggested that more than one airway generation should be examined in order to maximise the statistical power to detect a difference between study groups.

This is a timely paper that addresses an important issue in tissue analyses of inflammatory cells in chronic airway disease. I have seen many papers in the last few years that examine the effect of anti-inflammatory drugs using small numbers of patients and limited numbers of biopsies and sections. The quality of these papers has been variable and, in some cases, weak conclusions were drawn. This was due to a number of factors, including pressure to publish, limited resources and lack of experience in the analysis of immunoreactive cells. Adequate knowledge of the pathology of COPD and statistics is essential for developing a proper design and interpreting the results.

It is now clear that infiltration of the airway wall with chronic inflammatory cells is a feature of COPD. Most of the pathological changes in COPD are observed in the small airways and lung parenchyma [2,3]. However, it is extremely difficult to justify the sampling of small airways and lung parenchyma of patients for clinical research.

During the last few years, it has been shown that pathological changes can also be observed in large airways, but to a lesser extent. Researchers have used endobronchial biopsies to study

CORRESPONDENCE: Q. Hamid, James McGill Professor of Medicine and Pathology, Strauss Chair in Respiratory Medicine, Meakins Christie Labs, McGill University, Montreal, Quebec H2X 2P2, Canada. Fax: 1 5143987483. E-mail: qutayba.hamid@mcgill.ca the distribution of inflammatory cells, cytokine gene expression and response to treatment $[4,5]$. A number of inflammatory cells have been reported to be increased in the bronchial biopsies of COPD patients. The most prominent cells present are CD8+ cells. These cells are increased in the submucosal and epithelial layers. CD68+ macrophages are also increased in COPD, but the difference in the numbers of these cells between normal subjects and COPD patients is not as significant as CD8+ cells. Although neutrophils are considered to be a central cell in the pathogenesis of COPD, particularly at the early stage, their number in bronchial biopsies in COPD patients does not always increase. The contribution of $\mathrm{CD} 4+$, mast cells and basophils to COPD pathogenesis is uncertain. Remodelling is also a feature of COPD and changes in the epithelium are often seen; these are represented by thickening of the epithelium and metaplastic changes. Remodelling can also manifest itself through an increase in smooth muscle mass and mucous gland hyperplasia. Infiltration of inflammatory cells among the structural cells is not an uncommon feature in COPD and their potential contribution to the pathogenesis of COPD cannot be ignored.

However, most of the studies performed so far, including the study by GAMBLE et al. [1], have failed to address the changes in the number of inflammatory cells within smooth muscle and the epithelial layer. These areas were excluded from the surface areas of the biopsies when morphometry was performed. Although it might be helpful to avoid problems associated with loss of epithelium and absence of smooth muscle bundles in some biopsies, it is important that this issue is re-examined in future studies. This is particularly important when studying the effect of inhaled drugs that have a direct effect on these structural cells.

A number of cytokines and chemokines were described to be increased in COPD. These observations were based mostly on counting the number of a certain type of inflammatory cell expressing messenger RNA or immunoreactivity in the submucosal layer. The cytokines in question include interleukin (IL)-8, tumour necrosis factor (TNF)- $\alpha$ and interferoninducible protein $10[6,7]$, and epithelial and smooth muscle cells are a major source of these. In most studies published, including the study by GAMBLE et al. [1], the expression of these cytokines was only evaluated in inflammatory cells using immunocytochemistry. This might be due to the fact that it is easy to count the inflammatory cells within a specific area, but it is difficult to evaluate cytokine expression in structural cells. Production of IL- 8 and TNF- $\alpha$ by structural cells is more 
important than by inflammatory cells, and the anti-inflammatory effects of certain drugs can be cell specific and may fail to suppress cytokine expression by structural cells. Any evaluation of cytokine or chemokine expression that excludes structural cells is, therefore, incomplete.

It is also important to discuss the effect of the type of antibody used and the method of detection on the variability in the number of cells counted. It has been shown that there may be discrepancies between the results obtained by immunocytochemistry and in situ hybridisation. These techniques differ in their sensitivity, specificity and reproducibility. Eosinophils and eosinophil-associated cytokines were reported to be involved in the pathogenesis of COPD and were associated with exacerbations. Whether there is an increase in IL-5, eotaxin and monocyte chemotactic peptide- 4 in COPD biopsies remains to be confirmed.

The paper by GAMBLE et al. [1] addresses the appropriate use of statistically sound design to arrive at a conclusion. It describes microscopic measurements that are based on multilevel sampling, which includes subjects, airway, biopsy, blocks within each site and slides within each block. This is a wellknown design used in microscopy and statistical literature, but, unfortunately, not in clinical literature. The paper by GAMBLE et al. [1] raises the concern that a frequently used methodology for cell counting is not as simple as one may think, but indeed involves much more complexity. The simple tests frequently used in the medical literature may be useful for the final analyses of a study but are not adequate in designing a study, particularly those involving the use of a drug and a complex sampling procedure.

The authors chose to fix the number of repetitions at one for all the levels and evaluated the sample size required for a $50 \%$ effect of the treatment (or setting the time level at two for the within-group design). The size of the sample could be significantly affected by the number of blocks, the number of slides evaluated from each block and the number of biopsies per subject. Investigators should also consider the available resources when designing trials and decide on the most efficient design [8].

False replication was not considered in GAMBLE et al. [1] methodological paper $[9,10]$. However, false replication should be considered because it occurs when the sample size is taken as the total number of fields measured. This might produce a falsely significant test, leading to an unfounded conclusion. The real sample size is the number of subjects studied or, in this case, twice that, as bronchoscopic biopsies were obtained from two sites. In their conclusion, GAMBLE et al. [1] mention the paradigm of GUNDERSON and OSTERBY [11]: "do more less well". This is logical, but it should be interpreted with the acknowledgement that biological materials are heterogeneous, not only between subjects, but also within subjects.

In conclusion, the paper by GAMBLE et al. [1] is an interesting and important paper. More papers in this area need to be submitted to and accepted by respiratory and allergy journals. The results of the paper should be taken seriously when designing a metacentre study that examines the effect of drugs in chronic obstructive pulmonary disease. However, it will be difficult to apply these results to a single-centre study which examines a specific hypothesis or the role of a cell or a mediator. Performing 47 biopsies in each group in one centre is difficult and extremely demanding. Thus, the publishing of guidelines based on a consensus of an international group for obtaining, processing and analysing biopsies from chronic obstructive pulmonary disease patients would be very helpful.

\section{REFERENCES}

1 Gamble E, Qiu Y, Wang D, et al. Variability of bronchial inflammation in chronic obstructive pulmonary disease: implications for study design. Eur Respir J 2006; 27: 293-299.

2 Hogg JC. Pathophysiology of airflow obstruction in chronic obstructive pulmonary disease. Lancet 2004; 364: 709-721.

3 Saetta M, Baraldo S, Corbino L, et al. CD8 ${ }^{+}$cells in the lungs of smokers with chronic obstructive pulmonary disease. Am J Respir Crit Care Med 1999; 160: 711-717.

4 O'Shaughnessy T, Ansari TW, Barnes NC, Jeffery PK. Inflammation in bronchial biopsies of subjects with chronic bronchitis: inverse relationship of $\mathrm{CD}^{+} \mathrm{T}$ lymphocytes with FEV1. Am J Resp Crit Care Med 1997; 155: 852-857.

5 Gamble E, Grootendorst DC, Brighling CE, et al. Antiinflammatory effects of the phosphodiesterase- 4 inhibitor Cilomilast (Airflo) in chronic obstructive pulmonary disease. Am J Respir Crit Care Med 2003; 168: 976-982.

6 Miotto D, Christodoulopoulos P, Olivenstein R, et al. Expression of IFN-gamma-inducible protein; monocytes chemotactic proteins 1, 3, and 4; and eotaxin in TH1-and TH2 mediated lung diseases. J Allergy Clin Immunol. 2001; 107: 664-670.

7 Panzner P, Lafitte JJ, Tsicopoulos A, Hamid Q, Tulic MK. Marked up-regulation of T lymphocytes and expression of interleukin-9 in bronchial biopsies from patients with chronic bronchitis with obstruction. Chest 2003; 124: 1909-1915.

8 Howard CV, Reed MG. Statistics for Stereologists. In: Unbiased Stereology: Three-Dimensional Measurement in Microscopy. New York, Springer-Verlag, 1998; pp. 151176.

9 Baddeley A, Vedal Jensen EB. Design of Stereological Experiments. In: Stereology for Statisticians. New York, Chapman \& Hall, 2005; pp. 277-297.

10 Baddeley A, Vedal Jensen EB. Variance of Stereological Estimators. In: Stereology for Statisticians. New York, Chapman \& Hall, 2005; pp. 295-328.

11 Gundersen HJ, Osterby R. Optimizing sampling efficiency of stereological studies in biology or "do more less well!". J Microsc 1981; 121: 65-73. 